\title{
Rotationally resolved IR spectroscopy of hexamethylenetetramine (HMT) $\mathrm{C}_{6} \mathrm{~N}_{4} \mathrm{H}_{12}$
}

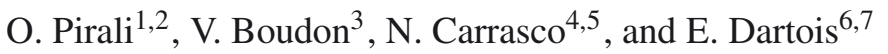 \\ ${ }^{1}$ Institut des Sciences Moléculaires d'Orsay, UMR 8214 CNRS-Université Paris-Sud, 91405 Orsay Cedex, France \\ 2 AILES beamline, Synchrotron SOLEIL Saint aubin, 91140 Gif-sur-Yvette, France \\ e-mail: olivier.pirali@synchrotron-soleil.fr \\ 3 Laboratoire Interdisciplinaire Carnot de Bourgogne, UMR 6303 CNRS-Université de Bourgogne, 9 Av. A. Savary, \\ BP 47870, 21078 Dijon Cedex, France \\ e-mail: Vincent. Boudon@u-bourgogne.fr \\ ${ }^{4}$ Université Versailles St-Quentin, UPMC Univ. Paris 06, CNRS, LATMOS, 11 blvd d'Alembert, 78280 Guyancourt, France \\ e-mail: Nathalie.Carrasco@latmos.ipsl.fr \\ 5 Institut Universitaire de France, France \\ ${ }^{6}$ CNRS-INSU, Institut d'Astrophysique Spatiale, UMR 8617, 91405 Orsay, France \\ e-mail: emmanuel.dartois@u-psud.fr \\ 7 Université Paris Sud, Institut d'Astrophysique Spatiale, UMR 8617, bâtiment 121, 91405 Orsay, France
}

Received 13 September 2013 / Accepted 19 November 2013

\begin{abstract}
Context. Hexamethylenetetramine (HMT) appears to be a potential constituent of several objects in space, including comets or Titan's atmosphere and, as an organic residue of ice irradiation in the laboratory, it may be present in the interstellar medium.

Aims. We performed a laboratory study of rotationally resolved intense IR bands of HMT to provide accurate line positions and synthetic spectra to be used for potential astronomical detections.

Methods. We used synchrotron-based high-resolution Fourier transform infrared spectroscopy to record the experimental data. A formalism and programs dedicated to the assignment, analysis, and simulation of absorption spectra of tetrahedral molecules were used to exploit the spectra.

Results. Infrared spectra of gas phase HMT were recorded and accurate wavenumbers and molecular parameters for four intense bands located in the $1000-1500 \mathrm{~cm}^{-1}$ spectral range suitable for astronomical searches were derived.
\end{abstract}

Key words. molecular data - planets and satellites: atmospheres - ISM: molecules

\section{Introduction}

Recent technical developments have made it feasible to record rotationally resolved infrared (IR) spectra of relatively large C-bearing molecules of astrophysical interest to provide accurate line positions for active search of such species in space (see, e.g., Brumfield et al. 2012; Albert et al. 2011; Pirali et al. 2012, 2013). In this context, hexamethylenetetramine (HMT), $\mathrm{C}_{6} \mathrm{~N}_{4} \mathrm{H}_{12}$, is of fundamental interest in astrochemistry. The molecule HMT is suspected to be present in various astrophysical media, all involving a N-rich photochemistry, such as interstellar dense clouds, or planetary atmospheric systems.

In interstellar dense clouds, interstellar grains acquire an ice mantle that is subsequently subjected to cosmic rays and an internal UV field. It has been shown that such photoprocessing of ices on grain surfaces can be simulated experimentally. In the laboratory, an ice layer whose chemical composition is analog to observations is deposited on a cooled substrate window placed in a high vacuum, while being irradiated by ultraviolet photons generated with an $\mathrm{H}_{2}$ plasma discharge lamp. To simulate the organic evolution of ice mantles, e.g., when the nascent stellar front progressively penetrates into the previously thermally cold and shielded parental cloud, the sample is slowly warmed up to analyze the residue containing less (but still) volatile products such as HMT. The refractory organic material produced under different conditions with respect to the ice mixture, UV spectrum, and dose, as well as the sample temperature, lead to the formation of HMT, as monitored by Fourier transform IR spectra displaying two specific peaks around 1230 and $1010 \mathrm{~cm}^{-1}$ (Muñoz Caro \& Schutte 2003), corresponding to the $v_{21}$ and $v_{22}$ modes, respectively. This species was first reported by Briggs et al. (1992) in such organic residues. Some evidence is presented for the formation of HMT at room temperature by Muñoz Caro et al. (2004), along with the important role of $\mathrm{H}_{2} \mathrm{O}$ ice as a catalyst for the formation of complex organic molecules. This species might also be present in the interstellar medium (ISM) and form part of comets. The molecule HMT can be formed, among the other refractory organic components, through the impact of energetic photons or cosmic rays on the ices, or thermal activated processes, as some laboratory studies have shown (e.g., Briggs et al. 1992; Bernstein et al. 1995; Cottin et al. 2001; Schutte 2002; Muñoz Caro et al. 2004; Vinogradoff et al. 2013 and references therein). The molecule HMT is among the major components of UV produced organic refractory residue when methanol is one ice component. A smaller amount seems to be produced when the mixture contains $\mathrm{CO}$ (and no $\mathrm{CH}_{3} \mathrm{OH}$ ) as carbon precursor source. Using an $\mathrm{H}_{2} \mathrm{O}: \mathrm{NH}_{3}: \mathrm{CH}_{3} \mathrm{OH}: \mathrm{CO}: \mathrm{CO}_{2}=2: 1: 1: 1: 1$ ice irradiation 


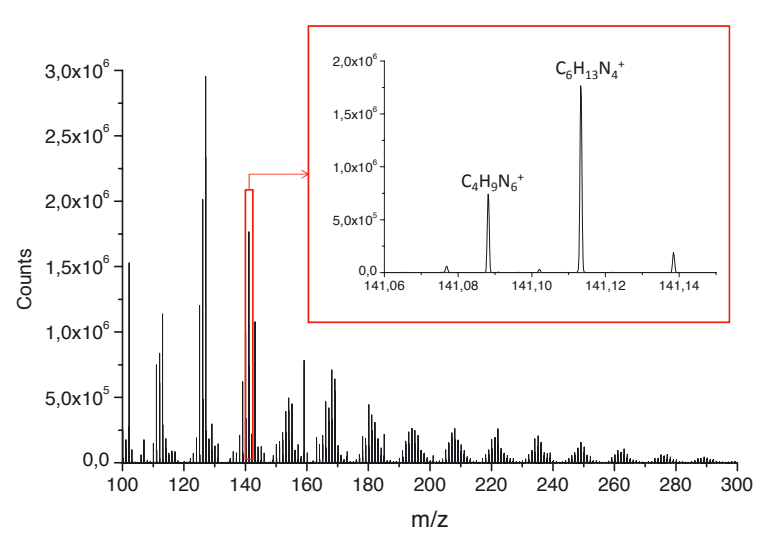

Fig. 1. High-resolution mass spectrum in the $50-500 \mathrm{~m} / \mathrm{z}$ range of an analog of Titan's atmospheric aerosol. As the injected neutral species are softly singly protonated during the electrospray ionization step, the ion detected corresponds to a $[\mathrm{M}+\mathrm{H}]^{+}$ion, with $\mathrm{M}$ as the native neutral. A zoom on the $141.0-141.2 \mathrm{~m} / \mathrm{z}$ range is given in the insert. The main ion detected at $m / z=141.11$ is consistent with the protonated form of HMT.

experiment, Muñoz Caro \& Dartois (2013) determined that the contribution of the $\mathrm{CO}$ and $\mathrm{CO}_{2}$ ice components to HMT formation in the organic residue, when compared to the contribution of the $\mathrm{CH}_{3} \mathrm{OH}$ ice component, was around $25 \%$. The spectra of ion-irradiated $\mathrm{H}_{2} \mathrm{O}: \mathrm{NH}_{3}: \mathrm{CO}$ ice mixture, when warmed up to room temperature, contain several bands that are tentatively assigned to HMT by Pilling et al. (2010). Following Muñoz Caro \& Schutte (2003), $\mathrm{NH}_{4}^{+}$might be one of the key species involved in the formation of highly complex molecules such as HMT, observed in experiments involving UV photoprocessing of interstellar ammonia-containing ice analogs. However, the presence of HMT molecules formed in the organic residue produced by the bombardments of interstellar ice analogs by energetic and heavy ions, has yet to be confirmed. Bernstein et al. (1994) presented the results of ultraviolet photolysis of matrix-isolated HMT and HMT frozen in $\mathrm{H}_{2} \mathrm{O}$ ice, and discussed the implications for infrared astronomy and astrochemistry. The low photostability of HMT when liberated into an open space environment may hamper its direct detection in astrophysics and the present study provides the spectroscopic parameters necessary for its search with high-resolution generation instruments in the infrared.

Moreover, HMT is a potential constituent of planetary atmospheres such as Titan's. First, it has been detected as a significant constituent of laboratory analogs mimicking Titan's atmospheric aerosols. Those analogs have been produced by plasma discharges in gas mixtures containing $98 \% \mathrm{~N}_{2}$ and $2 \%$ $\mathrm{CH}_{4}$. They are complex organic materials as illustrated by highresolution mass spectrometry in Fig. 1 by Pernot et al. (2010). The insert in Fig. 1 shows that the main ion at $m / z 141$ is $\mathrm{C}_{6} \mathrm{H}_{13} \mathrm{~N}_{4}^{+}$consistent with protonated HMT. The HMT structure has been confirmed by nuclear magnetic resonance (NMR) analysis (He et al. 2012). Secondly, HMT chemical synthesis occurring in Titan's aerosols analogs is different from the one occurring in ice-irradiation experiments designed for simulating ISM or cometary environments. In Titan's atmosphere, aerosols are bathed in a reactive gas phase providing precursors for HMT synthesis at the surface of the grains. Methyleneimine $\mathrm{CH}_{2} \mathrm{NH}$, the most plausible precursor for HMT synthesis, has been detected in large amounts in the gas phase surrounding, respectively, Titan's aerosols by Yelle \& al. (2010) and

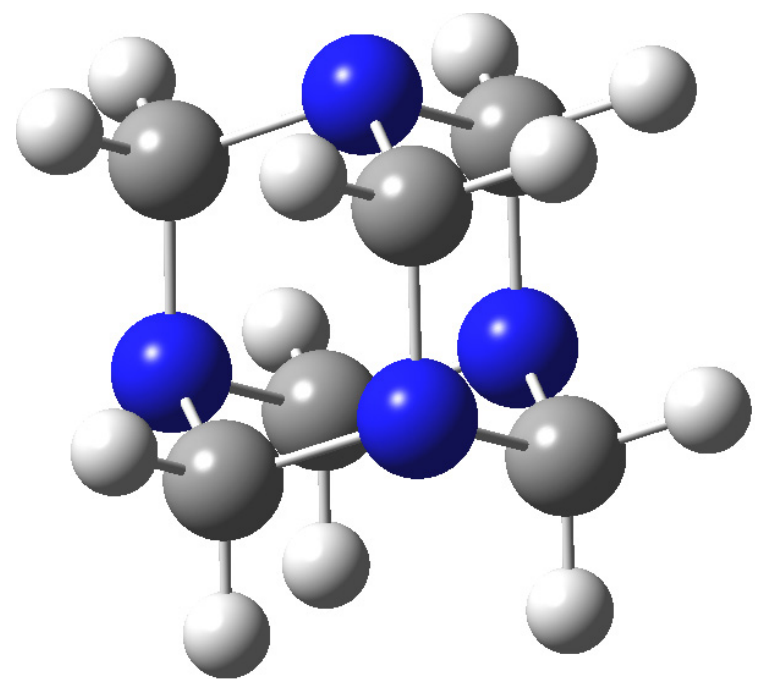

Fig. 2. The structure of the HMT molecule. Nitrogen atoms are represented in blue, carbon atoms in dark gray, and hydrogen atoms in light gray.

in their lab analogs by Carrasco et al. (2012). This clear detection of methyleneimine in Titan's atmosphere and in its laboratory simulation unravels a difficulty highlighted by Vinogradoff et al. (2012) for HMT synthesis. Indeed, the production of methyleneimine from $\mathrm{H}_{2} \mathrm{CO} / \mathrm{NH}_{3}$ ices requires overcoming an energy barrier and methyleneimine has never been directly observed as an intermediate in the ice-irradiation experiments. The clear detection of methyleneimine in Titan's atmosphere, combined with the important contribution of HMT to Titan's aerosols analogs, strongly support the idea that HMT may participate in the balance of heavy N-rich organic compounds in Titan's atmosphere.

As shown on Fig. 2, HMT has a symmetric tetrahedral cage-like structure, similar to adamantane $\mathrm{C}_{10} \mathrm{H}_{16}$, whose four $\mathrm{CH}$ corners are replaced by nitrogen atoms and whose edges remain methylene groups. This highly symmetric molecule has no permanent dipole moment preventing its radioastronomical search in the gas phase.

In the past, several laboratory studies reported spectroscopic information about HMT. In particular, the vibrational assignment of the IR spectrum was obtained thanks to condensed phase spectroscopy of HMT $\mathrm{H}_{12}$ and HMT $\mathrm{D}_{12}$ by Bertie \& Solinas (1974). Out of the 60 vibrational modes (25 distinct frequencies), only nine modes (involving upper states with triply degenerate $F_{2}$ symmetry) are infrared active from the ground vibrational state (GS, nondegenerate state of $A_{1}$ symmetry). Eight of them are displayed in Fig. 3. The low frequency $v_{25}-0$ fundamental band, which appears to be extremely weak, could not be recorded under the present experimental conditions. Four of the IR active bands, located in the $1000-1500 \mathrm{~cm}^{-1}$ spectral range, show relatively large absolute intensities: two $\mathrm{CN}$ stretching modes $\left(v_{22}\right.$ and $v_{21}$ located at about $1014 \mathrm{~cm}^{-1}$ and $\left.1240 \mathrm{~cm}^{-1}\right)$, the $\mathrm{CH}_{2}$ wagging, and the $\mathrm{CH}_{2}$ deformations modes $\left(v_{20}\right.$ at $1362 \mathrm{~cm}^{-1}$ and $v_{19}$ at $1463 \mathrm{~cm}^{-1}$ ). The aim of the paper is to perform the analysis of the rotational structure for these four vibrational bands of HMT, which may be used for an astronomical search of this molecule in the gas phase.

The paper is organized as follows. The experiment and analysis are described in Sect. 2, the results and discussion compose Sect. 3, and the last section contains the conclusions and perspectives revealed by this study. 
O. Pirali et al.: Rotationally resolved IR spectroscopy of hexamethylenetetramine (HMT) $\mathrm{C}_{6} \mathrm{~N}_{4} \mathrm{H}_{12}$

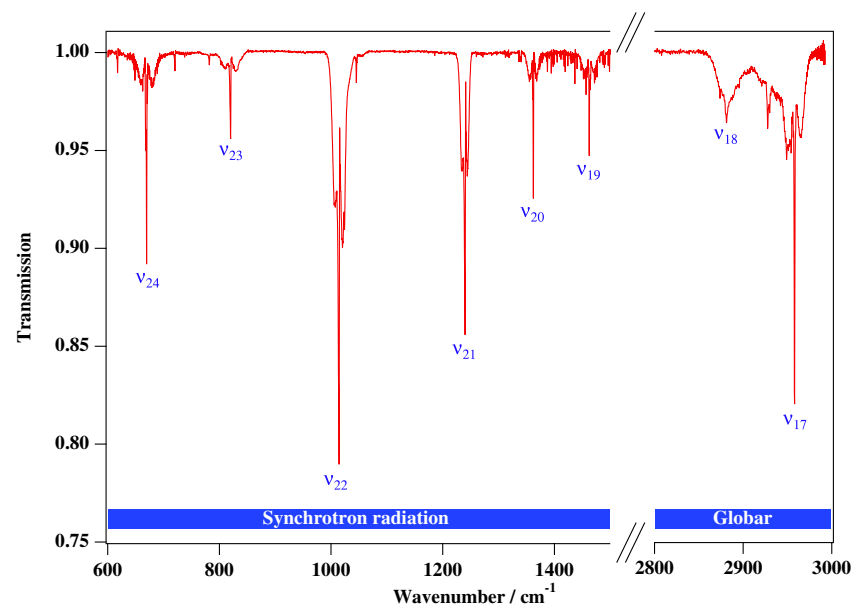

Fig. 3. Low-resolution gas phase IR spectrum of HMT. The light source used to study the different spectral regions is indicated.

\section{Methods}

\subsection{Experiment}

We recorded all spectra in the $33-4500 \mathrm{~cm}^{-1}$ range using a highresolution Bruker IFS 125 interferometer located at the AILES beamline of the SOLEIL synchrotron facility. We injected gas phase HMT (99.5\% from Aldrich) in a multipass cell of which the optics (White-type arrangement) were set to obtain about $151.8 \mathrm{~m}$ absorption pathlength. The $\mathrm{ZnSe}$ windows $\left(0.5^{\circ}\right.$ wedge, $5 \mathrm{~mm}$ thickness) separated the cell from the interferometer. We recorded the spectra with a spectral resolution of $0.001 \mathrm{~cm}^{-1}$ and we have made use of the SOLEIL synchrotron far-IR radiation extracted by the AILES beamline as the continuum source of the FT interferometer (Brubach et al. 2010). We injected the maximum vapor pressure attainable at room temperature (about $0.001 \mathrm{mbar}$ ) to detect and rotationally resolve the IR bands.

The peak finder available in the Bruker OPUS software was used and line positions were calibrated using lines of the $v_{2}$ band of water (Rothman et al. 2009). We chose a fixed experimental uncertainty of $0.0002 \mathrm{~cm}^{-1}$, which is a reasonable compromise for the four bands studied in this work.

\subsection{General theoretical considerations}

For the $F_{2}$ symmetry upper vibrational states of the triply degenerate fundamental bands, Coriolis forces induced by molecular rotation lift the degeneracy, causing a splitting into three components denoted as $F_{2}^{(+)}(J), F_{2}^{(0)}(J)$, and $F_{2}^{(-)}(J)$ (see Herzberg 1945 , for a general description of the rotational energy levels of spherical tops). For the $F_{2}-A_{1}$ vibrational transitions considered in this work, selection rules apply such that $F^{(-)}(J), F^{(0)}(J)$, and $F^{(+)}(J)$ states are reached by $\Delta J=+1,0$ and -1 transitions, respectively. Rotational levels possess $A_{(1 \text { or } 2)}, E$, or $F_{(1 \text { or } 2)}$ symmetries and the $A-A, E-E$, and $F-F$ selection rules apply. When higher order rotation-vibration couplings are not considered, the various symmetries for a given $J$ value (rotational quantum number) are degenerate, which yields $P$ - and $R$-branches consisting of single lines. When second- and higher-order interactions become larger (Coriolis interactions or centrifugal distortion effects), the energies of the rovibrational levels of a given $J$ value split, which leads to the so-called "tetrahedral structure" for every rotational transition in each of the branches. It should be noted that for fundamental bands of spherical top molecules, in the absence of strong perturbations, the selection rules are
Table 1. Observed and calculated wavenumbers with their assignments of the IR active fundamental modes of HMT.

\begin{tabular}{lccc}
\hline \hline & \multicolumn{2}{c}{ Wavenumbers/cm ${ }^{-1}$} & \\
Assignment & Obs. & Calc. & Intensity $/ \mathrm{km} \mathrm{mol}^{-1}$ \\
\hline$v_{25}$ & & 487.9 & 1.5 \\
$v_{24}$ & $669.2^{b}$ & 668.8 & 23 \\
$v_{23}$ & $817.8^{a}$ & 831.2 & 17 \\
$v_{22}$ & $1014.4^{a}$ & 1020.5 & 143 \\
$v_{21}$ & $1240.3^{a}$ & 1252.4 & 61 \\
$v_{20}$ & $1362.5^{a}$ & 1378.5 & 15 \\
$v_{19}$ & $1463.0^{a}$ & 1484.6 & 17 \\
$v_{18}$ & $2881.1^{b}$ & 3075.2 & 105 \\
$v_{17}$ & $2957.2^{b}$ & 3126.2 & 57 \\
\hline
\end{tabular}

Notes. ${ }^{(a)}$ The values were obtained from the rotational analysis and fit (see the text below), ${ }^{(b)}$ indicates those obtained from the maximum absorption. The last column lists integrated band intensities as obtained through the DFT calculation.

such that each upper state rovibrational level is reached by only one transition from the ground vibrational state. Therefore, combination differences cannot be determined, so that it is impossible to simultaneously fit lower and upper state parameters.

\subsection{Density functional theory (DFT) calculations}

Because of the impossibility to experimentally determine the GS rotational parameters, we performed harmonic DFT calculations at the B97-1//ANO-DZP level using Gaussian09 software (Roos et al. 2005). This method seems to lead to relatively accurate rotational parameters (Pirali et al. 2013). The parameter $B_{0}$ was thus estimated equal to about $1.87215 \mathrm{GHz}$ $\left(0.0624482 \mathrm{~cm}^{-1}\right)$ and fixed to this value in the following. The same DFT calculations gave us estimates for the fundamental integrated band intensities. Table 1 provides the wavenumbers and assignment of the nine IR active modes of HMT.

\subsection{Assignment and analysis of the spectra}

For the analysis of the rotational structure of the IR bands of HMT, we followed the same approach that was developed in the case of adamantane by Pirali et al. (2012). Line assignments were performed thanks to the SPVIEW software (Wenger et al. 2008). For spectrum calculations and fits, we used the XTDS (Wenger et al. 2008) software that implements the Dijon tensorial model for spherical-top molecules of tetrahedral symmetry. Each band was considered isolated. The ground state effective Hamiltonian was expanded at zeroth order, resulting in only one molecular constant in this case, which is $B_{0}$. The value of $B_{0}$ was fixed to the DFT value given in Sect. 2.3. The $v_{i}=1(i=22$, 21, 20 and 19) upper vibrational state effective Hamiltonian was expanded up to the fifth order, resulting in 17 parameters. The dipole moment operator itself was, in each case, expanded only up to the zeroth order. The single dipole moment parameter was arbitrarily fixed at 1 , since we do not have absolute intensity measurements here.

We started the analysis using an approximate band center value and varying by hand the Coriolis and a few other low order parameters until we obtained a reasonable spectrum simulation, allowing line assignments. Once assigned, we fitted the line positions using a nonlinear least-squares procedure of LevenbergMarquardt type. We performed several assignments and fit steps. Table 2 shows the results of the fit for the $v_{22}-\mathrm{GS}, v_{21}-\mathrm{GS}$, 
Table 2. Effective Hamiltonian parameters.

\begin{tabular}{|c|c|c|c|c|c|}
\hline $\begin{array}{l}\text { Parameter } \\
\Omega(K, n C)\end{array}$ & $v_{22}$ & $v_{21}$ & $v_{20}$ & $v_{19}$ & $\begin{array}{l}\text { Notation from } \\
\text { Robiette et al. (1976) }\end{array}$ \\
\hline $0\left(0,0 A_{1}\right)$ & 1014.832075(41) & $1240.650349(41)$ & $1362.500145(45)$ & $1462.363744(47)$ & $v_{i}$ \\
\hline $1\left(1,0 F_{1}\right)$ & $1.84297(28) \times 10^{-2}$ & $8.91601(20) \times 10^{-2}$ & $3.37902(28) \times 10^{-2}$ & $-3.10933(30) \times 10^{-2}$ & $3 \sqrt{2}(B \zeta)_{i}$ \\
\hline $2\left(0,0 A_{1}\right)$ & $-5.9745(37) \times 10^{-5}$ & $-3.8685(18) \times 10^{-5}$ & $-4.2321(33) \times 10^{-5}$ & $1.5008(17) \times 10^{-5}$ & $B_{i}-B_{0}$ \\
\hline $2(2,0 E)$ & $7.0941(82) \times 10^{-5}$ & $-2.2745(35) \times 10^{-5}$ & $1.6829(50) \times 10^{-5}$ & $-2.270(43) \times 10^{-6}$ & $-(1 / 2) \alpha_{220}^{i}-6 \alpha_{224}^{i}$ \\
\hline $2\left(2,0 F_{2}\right)$ & $-4.7516(90) \times 10^{-5}$ & $1.3 \times 10^{-5 \dagger}$ & $-1.9726(64) \times 10^{-5}$ & $7.0 \times 10^{-6 \dagger}$ & $-(3 / 4) \alpha_{220}^{i}+6 \alpha_{224}^{i}$ \\
\hline $3\left(1,0 F_{1}\right)$ & $7.789(68) \times 10^{-8}$ & $2.126(24) \times 10^{-8}$ & $2.413(38) \times 10^{-8}$ & $-3.369(26) \times 10^{-8}$ & $-(3 \sqrt{3} / 4 \sqrt{2}) F_{110}^{i}$ \\
\hline $3\left(3,0 F_{1}\right)$ & $7.528(42) \times 10^{-8}$ & $1.412(18) \times 10^{-8}$ & $9.26(24) \times 10^{-9}$ & $4.04(20) \times 10^{-9}$ & $(3 \sqrt{5} / 2 \sqrt{2}) F_{134}^{i}$ \\
\hline $4\left(0,0 A_{1}\right)$ & $-1.886(80) \times 10^{-10}$ & & & & $-\left(D_{4}-D_{0}\right)$ \\
\hline $4(2,0 E)$ & $2.660(61) \times 10^{-10}$ & & & & $\sqrt{3} / 8 G_{220}+3 \sqrt{3} / 2 G_{224}^{i}$ \\
\hline $4\left(2,0 F_{2}\right)$ & $-4.0(1.1) \times 10^{-11}$ & & & & $3 \sqrt{3} / 16 G_{220}^{i}-3 \sqrt{3} / 2 G_{224}^{i}$ \\
\hline $4\left(4,0 A_{1}\right)$ & $8.7(1.1) \times 10^{-12}$ & & & & $-3 \sqrt{5} / 4 \sqrt{2}\left(D_{i t}-D_{0 t}\right)$ \\
\hline $4(4,0 E)$ & $1.69(33) \times 10^{-11}$ & & & & $-3 \sqrt{5} / 4 \sqrt{2}\left(D_{i t}-D_{0 t}\right)$ \\
\hline $4\left(4,0 F_{2}\right)$ & $-6.68(31) \times 10^{-11}$ & & & & $-3 \sqrt{7} / 2 G_{244}^{i}+\sqrt{21} / 2 \sqrt{22} G_{246}^{i}$ \\
\hline $5\left(1,0 F_{1}\right)$ & $9.12(38) \times 10^{-13}$ & & & & \\
\hline Lines fitted & 5207 & 3411 & 2623 & 1224 & \\
\hline$J_{\max }$ & 92 & 82 & 70 & 86 & \\
\hline$d_{\mathrm{rms}} / 10^{-3} \mathrm{~cm}^{-1}$ & 0.297 & 0.371 & 0.442 & 0.698 & \\
\hline
\end{tabular}

Notes. Values are in $\mathrm{cm}^{-1}$. Nonfitted parameters are fixed to zero. The value $J_{\max }$ is the maximum $J$ value for assigned lines. Uncertainties in the last digits for each parameter are provided in parentheses; they represent $1 \sigma$ standard deviation obtained from the least-squares fit. ${ }^{(\dagger)}$ Fixed value.

$v_{20}-\mathrm{GS}$, and $v_{19}-\mathrm{GS}$ bands studied here. The next section discusses the results for each band.

\section{Results and discussion}

As already outlined, HMT has nine triply degenerate IR active modes ( $F_{2}$ symmetry) for which the calculated intensities range from about $1.5 \mathrm{~km} \mathrm{~mol}^{-1}$ to about $143 \mathrm{~km} \mathrm{~mol}^{-1}$. In the experimental spectrum taken at low resolution (see Fig. 3), eight IR active modes can be identified. Table 1 lists the frequencies of the eight vibrational band centers with their assignment. The $v_{25}$ (CNC deformation) IR active mode is too weak (calculated intensity of $1.5 \mathrm{~km} \mathrm{~mol}^{-1}$ ) to be observed in the gas phase with our experimental setup. In the following, we present high-resolution scans of the relatively intense vibrational bands located in the $1000-1500 \mathrm{~cm}^{-1}$ spectral range (namely $\left.v_{22}, v_{21}, v_{20}, v_{19}\right)$, the $\mathrm{CH}$ stretching modes $v_{18}$ and $v_{17}$ appearing as broad unresolved saturated features. The four rotationally resolved IR active modes under consideration show relatively different rotational patterns. Figure 4 shows an overview of the high-resolution spectra of the $v_{22}, v_{21}, v_{20}, v_{19}$ bands, compared to the simulations obtained from the fitted parameters (see Table 2).

The upper traces (in blue) are the predicted spectra (calculations at $300 \mathrm{~K}$ ) and the lower traces are the experimental spectra (in red). The residuals expressed in $10^{-3} \mathrm{~cm}^{-1}$ are indicated at the bottom of each panel. It should be noted that, because of the presence of several low-lying bending fundamental vibrational modes (e.g., modes located at $392 \mathrm{~cm}^{-1}, 472 \mathrm{~cm}^{-1}$, and $512 \mathrm{~cm}^{-1}$ ), our room temperature spectra exhibit many hot band lines. The most striking example of the presence of hot bands is visible in the $Q$ branch region of the $v_{21}$ band where a second intense $Q$ branch is detected. The present analysis is, however, restricted to the vibrational fundamental.

\subsection{The $\mathrm{CN}$ stretching modes $v_{22}$ and $v_{21}$ at $1014 \mathrm{~cm}^{-1}$ and $1240 \mathrm{~cm}^{-1}$}

The $v_{22}$ and $v_{21}$ bands are the most intense absorptions of HMT (calculated intensity of $143 \mathrm{~km} \mathrm{~mol}^{-1}$ and $61 \mathrm{~km} \mathrm{~mol}^{-1}$, respectively). Figure 5 shows parts of the $v_{22}$ spectrum in the $P$ and $R$ branches. In this part of the band, the splitting of the clusters of lines into multiple components is well resolved for each $J$ value. There is good agreement between the calculated and experimental spectrum both in terms of line frequencies and relative intensities, despite the presence of additional hot band lines in the experimental spectrum (which were not analyzed in the present work). As can be seen in the left panel of Fig. 6, the $Q$ branch of $v_{22}$ is also well resolved and many individual lines (observed on top of the broad unresolved absorption produced by the superposition of numerous lines involving all $J$ values) could be assigned in this dense spectral region.

As visible on the residuals from Fig. 4, the rotational structure of the $v_{21}$ band is also very well reproduced by the calculations, again despite the presence of hot bands in the spectrum.

\section{2. $\mathrm{CH}_{2}$ wagging and $\mathrm{CH}_{2}$ deformations modes ( $v_{20}$ at $1362 \mathrm{~cm}^{-1}$ and $v_{19}$ at $1463 \mathrm{~cm}^{-1}$ )}

The $v_{20}$ and $v_{19}$ bands are weaker than $v_{22}, v_{21}$ bands (their calculated intensities are $15 \mathrm{~km} \mathrm{~mol}^{-1}$ and $17 \mathrm{~km} \mathrm{~mol}^{-1}$, respectively). For the $v_{20}$ band, clusters of lines are well resolved in the $P$ and $R$ branches and few lines could be assigned in the $Q$ branch. A few intense absorptions from water lines are also observed. For the $v_{19}$ band the splitting of the different $J$ components is much smaller (see the zoom of the $R$ branch in Fig. 6) than for the other bands and no $Q$ branch line was assigned in this spectrum. As we are close to the $v_{2}$ band center of water, many $\mathrm{H}_{2} \mathrm{O}$ absorption lines are identified in this part of the spectrum.

\subsection{Detection limits in dense clouds}

One of the best place to search for HMT is the sublimation front of ice mantles in the region close to newly born stars in dense 
O. Pirali et al.: Rotationally resolved IR spectroscopy of hexamethylenetetramine (HMT) $\mathrm{C}_{6} \mathrm{~N}_{4} \mathrm{H}_{12}$
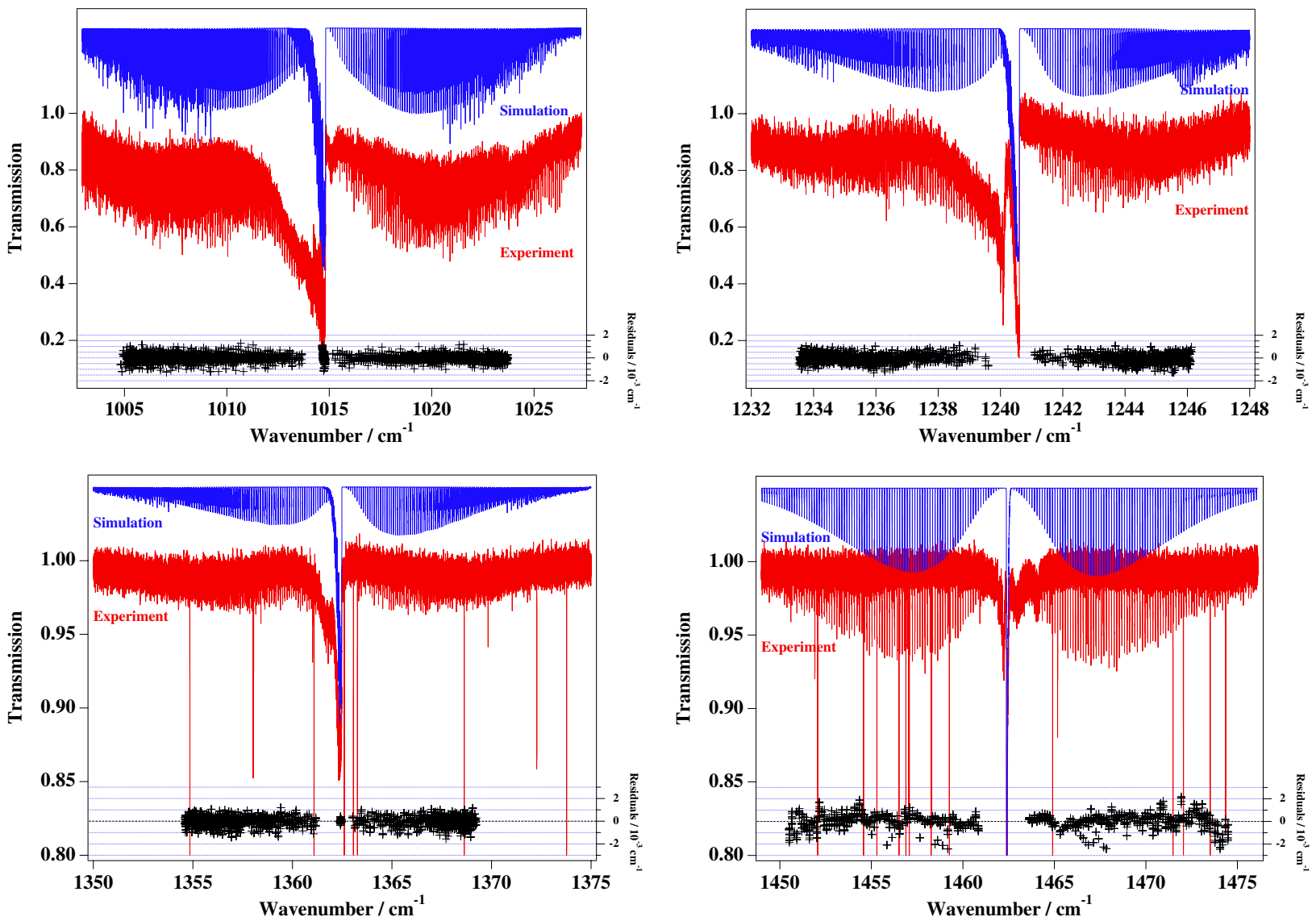

Fig. 4. Overview of the high-resolution spectra of the $v_{22}$ (upper left panel), $v_{21}$ (upper right), $v_{20}$ (lower left), and $v_{19}$ bands (lower right), compared to the simulations. Fit residuals for line positions are given (black crosses at the bottom of each panel).
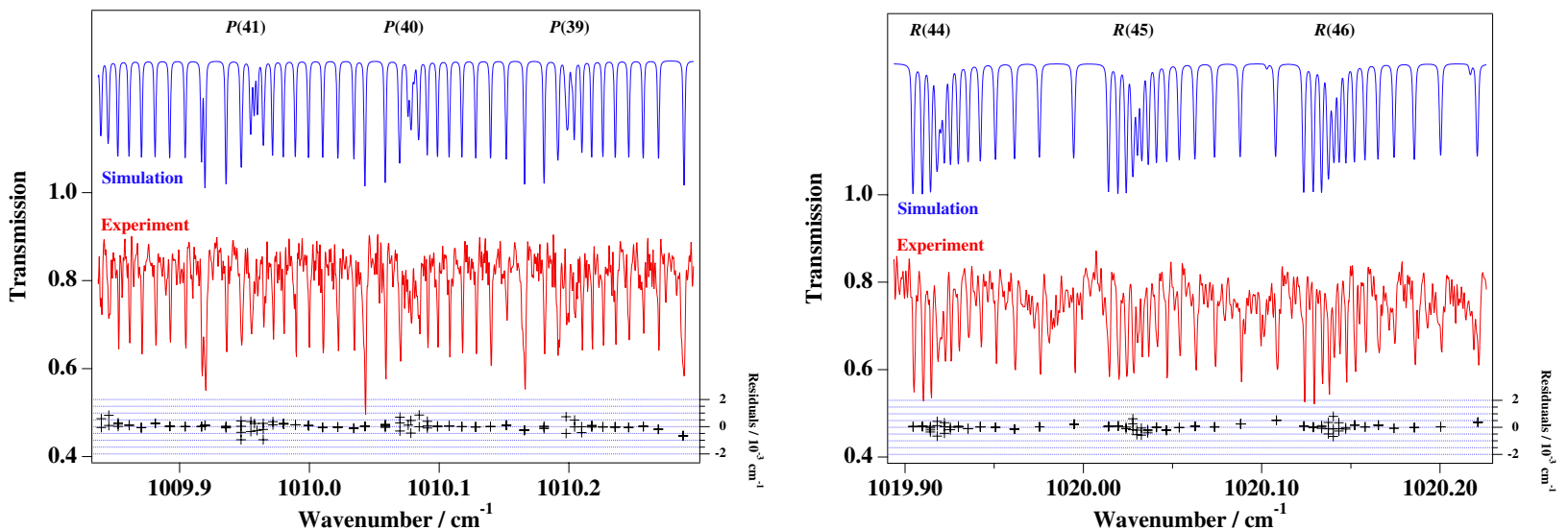

Fig. 5. Parts of the $P(l e f t)$ and $R($ right $)$ branches of the $v_{22}$ band of HMT, compared to the simulations. Fit residuals for line positions are given (black crosses at the bottom of each panel). Clusters of lines for each $J$ value are clearly resolved on the spectra.

clouds. The highest contrast for detection will arise because of the numerous lines forming the $Q$ branch.

The typical half widths of the $Q$ branches are a fraction of a wavenumber and thus require high-resolution observations. The maximum optical depth for resolved observations, for a given mode can be estimated as follows:

$\tau_{Q}(T) \approx n_{\mathrm{H}} \cdot f_{\mathrm{H}_{2} \mathrm{O}}^{\mathrm{ice}} \cdot f_{\mathrm{HMT}} \cdot \eta_{\mathrm{H}_{2} \mathrm{O}}^{\text {subl }} \cdot A_{Q}(T) / \Delta v_{1 / 2}$

where $f_{\mathrm{H}_{2} \mathrm{O}}^{\text {ice }}$ and $f_{\mathrm{HMT}}$ are the fraction of water ice mantle, and the fraction of the mantle converted to HMT, respectively. The parameter $\eta_{\mathrm{H}_{2} \mathrm{O}}^{\text {subl }}$ is the $\mathrm{H}_{2} \mathrm{O}$ gas to solid ratio, or the possibly maximum observed sublimated fraction.
$A_{Q}(\mathrm{~T})=\frac{\int_{\mathrm{Q}} \tau \mathrm{d} v}{\int_{\text {band }} \tau \mathrm{d} v} \cdot A_{\text {mode }}(\mathrm{T}) \approx \frac{1}{3} \cdot A_{\text {mode }}(\mathrm{T})$ is the intensity of the mode distributed in the $Q$ branch absorptions at a given temperature.

The strongest mode $\left(v_{22}\right)$ falls in the saturated part of the silicates absorption band, thus $v_{21}$ is probably the most favorable mode to focus on for detection, with $A_{\mathrm{Q}}\left(v_{21}\right) \approx 20 \mathrm{~km} \mathrm{~mol}^{-1}$. At $100 \mathrm{~K}$, the half width of the $v_{21} Q$ branch is about $0.08 \mathrm{~cm}^{-1}$.

For a molecular cloud with a visual extinction $A_{V}$ of 10 , $n_{\mathrm{H}} \approx 2 \times 10^{22} \mathrm{~cm}^{-2}, f_{\mathrm{H}_{2} \mathrm{O}}^{\text {ice }}$ can reach $10^{-4}$, and $\eta_{\mathrm{H}_{2} \mathrm{O}}^{\text {subl }}$ is highly variable with typical values $10^{-1}-10^{-4}$ from source to source 

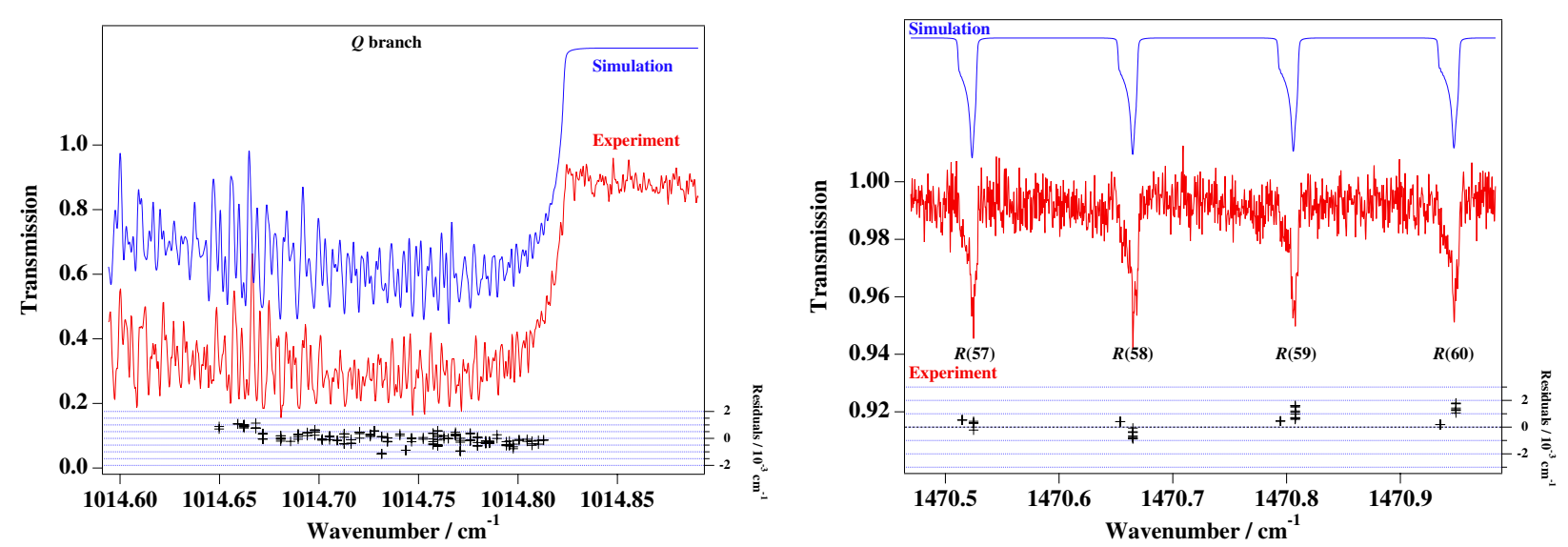

Fig. 6. Parts of the $Q$ branch of the $v_{22}$ band (left) and of the $R$ branches of the $v_{19}$ band (right) of HMT, compared to the simulations. Fit residuals for line positions are given (black crosses at the bottom of each panel). The agreement is again very clear, even in dense $Q$ branches or in unresolved line clusters.

(e.g., van Dishoeck \& Helmich 1996; Dartois et al. 1998; van Dishoeck et al. 2011; Coutens 2012).

From the laboratory side, the expected HMT abundances in UV photolysis production of the residues, starting with different ice mixtures, and UV doses of about $0.25 \mathrm{UV}$ photon per ice molecule was evaluated by Bernstein et al. (1995); Muñoz Caro $\&$ Schutte (2003). The fraction of carbon atoms present in the starting ice mixture converted to carbon atoms in HMT, is 5$7 \%$ for the standard ice mixtures and exceptionally about two times higher in specific mixtures. In terms of numbers of HMT molecules produced, given that simple interstellar ice mantles components typically have one carbon atom, it translates into at most 1 to $2 \%$ of the initial number of molecules prior to irradiation. This leads to $f_{\mathrm{HMT}} \approx 10^{-2}$.

For the $v_{21} Q$ branch, these numbers translate to:

$0.1>\tau_{\mathrm{Q}}\left(v_{21}\right) \gtrsim 1 \times 10^{-5}$

and are about two to three times higher for the $v_{18}$ and $v_{22}$ $Q$ branches, respectively. The large spread over several orders of magnitude is dominated by the $\mathrm{H}_{2} \mathrm{O}$ gas to solid ratio, i.e., the sublimated mantle fraction. These bands would possibly be detectable in the lines of sights of protostellar warm environments with the highest mantle sublimated fraction. The band contrast obtained with the gas phase $Q$ branches is probably the highest possible, the corresponding bands in the ices residues being much larger. In addition, HMT is formed from the products of the photolysis of ice mantles only when these ices are warmed up above about $200 \mathrm{~K}$. In the laboratory HMT has, up to now, never been detected at low temperatures, whatever the signal-tonoise ratio, in ice mantles simulations. This implies that the best place to look for HMT is in the inner parts of protostellar objects. In these regions, the temperature of the grains is high enough to sublimate the mantles $(100 \mathrm{~K}-300 \mathrm{~K})$ due to the propagation of a thermal front, but these regions are still protected from the central star photodissociating photons by the more refractory grains ( silicates, carbonaceous dust,...) closer to the star. Those conditions ensure that the destruction timescale for HMT is not too rapid with respect to the mantle sublimation front speed.

\section{Conclusions}

We performed high-resolution Fourier-transform spectroscopy of HMT in the gas phase for the first time. We reported the band center of eight IR active modes and the detailed rotational analysis of four of the most intense bands located in the $1000-1500 \mathrm{~cm}^{-1}$ spectral range: the $v_{22}$ and $v_{21} \mathrm{CN}$ stretching modes with respective band center at $1014.832075(41) \mathrm{cm}^{-1}$ and $1240.650349(41) \mathrm{cm}^{-1}$, the $v_{20}$ and $v_{19} \mathrm{CH}_{2}$ wagging and deformation modes, with respective band centers at $1362.500145(45) \mathrm{cm}^{-1}$ and $1462.363744(47) \mathrm{cm}^{-1}$. These relatively intense bands may be used for an infrared search for this molecule in the interstellar medium or in the atmosphere of Titan.

Acknowledgements. O.P. acknowledges the French national program "Physique Chimie du Milieu Interstellaire" for partial funding of this project. We also thank the GdR number 3152 "SpecMo" of the CNRS.

\section{References}

Bernstein, M. P., Sandford, S. A., Allamandola, L. J., \& Chang, S. 1994, J. Phys. Chem., 98, 12206

Bernstein, M. P., Sandford, S. A., Allamandola, L. J., Chang, S., \& Scharberg, M. A. 1995, ApJ, 454, 327

Bertie, J. E., \& Solinas, M. 1974, J. Chem. Phys., 61, 1666

Briggs, R., Ertem, G., Ferris, J. P., et al. 1992, Origins of Life and Evolution of the Biosphere, 22, 287

Brubach, J.-B., Manceron, L., Rouzière, M., et al. 2010, AIP Conf. Proc., 1214, 81

Carrasco, N., Gautier, T., Touhami Es-sebbar, E., Pernot, P., \& Cernogora, G. 2012, Icarus, 219, 230

He, C., Lin, G., \& Smith, M. A. 2012, Icarus, 220, 627

Muñoz Caro, G. M., \& Dartois, E. 2013, Chem. Soc. Rev., 42, 2173

Muñoz Caro, G. M., \& Schutte, W. A. 2003, A\&A, 412, 121

Muñoz Caro, G. M., Meierhenrich, U., Schutte, W. A., Thiemann, W. H.-P., \& Greenberg, J. M. 2004, A\&A, 413, 209

Pernot, P., Carrasco, N., Thissen, R., \& Schmitz-Afonso, I. 2010, Anal. Chem., 82,1371

Pilling, S., Duarte, E. S., da Silveira, E. F., et al. 2010, A\&A, 509, A87

Pirali, O., Boudon, V., Oomens, J., \& Vervloet, M. 2012, J. Chem. Phys., 136, 024310

Pirali, O., Goubet, M., Huet, T. R., et al. 2013, Phys. Chem. Chem. Phys., 15, 10141

Robiette, A., Gray, D., \& Birss, F. 1976, Mol. Phys., 32, 1591

Roos, B. O., Lindh, R., Malmqvist, P. A., Veryazov, V., \& Widmark, P. O. 2005, J. Phys. Chem. A, 109, 6575

Rothman, L. S., Gordon, I. E., Barbe, A., et al. 2009, J. Quant. Spectr. Rad. Transf., 110, 533

Vinogradoff, V., Rimola, A., Duvernay, F., et al. 2012, Phys. Chem. Chem. Phys., 14,12309

Wenger, C., Boudon, V., Rotger, M., Sanzharov, M., \& Champion, J.-P. 2008, J. Mol. Spectrosc., 251, 102

Yelle, R. V., Vuitton, V., Lavvas, P., et al. 2010, Faraday Discuss., 147, 31 